OPEN ACCESS

Edited by:

Zhen Yuan,

University of Macau, China

Reviewed by:

Jiaojian Wang,

University of Electronic Science and Technology of China, China

Sergei Anatolievich Zhivolupov, Kirov Military Medical

Academy, Russia

*Correspondence:

Chunqi Chang

cqchang@szu.edu.cn

Nizhuan Wang

wangnizhuan1120@gmail.com

tThese authors have contributed equally to this work

Specialty section:

This article was submitted to

Brain Imaging and Stimulation,

a section of the journal

Frontiers in Human Neuroscience

Received: 11 January 2020

Accepted: 13 May 2020

Published: 06 July 2020

Citation:

Wu H, Yan H, Yang Y, Xu M, Shi Y, Zeng W, Li J, Zhang J, Chang $C$ and

Wang N (2020) Occupational Neuroplasticity in the Human Brain: A Critical Review and Meta-Analysis of

Neuroimaging Studies.

Front. Hum. Neurosci. 14:215. doi: 10.3389/fnhum.2020.00215

\section{Occupational Neuroplasticity in the Human Brain: A Critical Review and Meta-Analysis of Neuroimaging Studies}

\author{
Huijun $\mathrm{Wu}^{1+}$, Hongjie Yan ${ }^{2 \dagger}$, Yang Yang ${ }^{3}$, Min $\mathrm{Xu}^{4}$, Yuhu Shi ${ }^{5}$, Weiming Zeng ${ }^{5}$, Jiewei $\mathrm{Li}^{6}$, \\ Jian Zhang ${ }^{7}$, Chunqi Chang ${ }^{1,8 *}$ and Nizhuan Wang ${ }^{9 *}$ \\ ${ }^{1}$ School of Biomedical Engineering, Health Science Center, Shenzhen University, Shenzhen, China, ${ }^{2}$ Department of \\ Neurology, Affiliated Lianyungang Hospital of Xuzhou Medical University, Lianyungang, China, ${ }^{3}$ Center for Brain Science and \\ Learning Difficulties, Institute of Psychology, Chinese Academy of Sciences, Beijing, China, ${ }^{4}$ Center for Brain Disorders and \\ Cognitive Science, Shenzhen University, Shenzhen, China, ${ }^{5}$ Lab of Digital Image and Intelligent Computation, Shanghai \\ Maritime University, Shanghai, China, ${ }^{6}$ Department of Electrical and Electronic Engineering, The University of Hong Kong, \\ Pokfulam, Hong Kong, ${ }^{7}$ School of Pharmacy, Health Science Center, Shenzhen University, Shenzhen, China, ${ }^{8}$ Pengcheng \\ Laboratory, Shenzhen, China, ${ }^{9}$ Artificial Intelligence \& Neuro-Informatics Engineering (ARINE) Laboratory, School of \\ Computer Engineering, Jiangsu Ocean University, Lianyungang, China
}

Many studies have revealed the structural or functional brain changes induced by occupational factors. However, it remains largely unknown how occupation-related connectivity shapes the brain. In this paper, we denote occupational neuroplasticity as the neuroplasticity that takes place to satisfy the occupational requirements by extensively professional training and to accommodate the long-term, professional work of daily life, and a critical review of occupational neuroplasticity related to the changes in brain structure and functional networks has been primarily presented. Furthermore, meta-analysis revealed a neurophysiological mechanism of occupational neuroplasticity caused by professional experience. This meta-analysis of functional neuroimaging studies showed that experts displayed stronger activation in the left precentral gyrus [Brodmann area (BA)6], left middle frontal gyrus (BA6), and right inferior frontal gyrus (BA9) than novices, while meta-analysis of structural studies suggested that experts had a greater gray matter volume in the bilateral superior temporal gyrus (BA22) and right putamen than novices. Together, these findings not only expand the current understanding of the common neurophysiological basis of occupational neuroplasticity across different occupations and highlight some possible targets for neural modulation of occupational neuroplasticity but also provide a new perspective for occupational science research.

Keywords: occupational science, neuroplasticity, occupational neuroplasticity, neuroimaging, meta-analysis

\section{INTRODUCTION}

One of the characteristics of the human brain is its lifelong reorganization of both structure and function (Pascual-Leone et al., 2005). The term "neuroplasticity" refers to changes in function or structure that occur in the brain to adapt to external and/or internal factors (Zilles, 1992; Kempermann, 2006). The extent of neuroplastic remodeling depends on the relevance of individual 
changes and may have beneficial or maladaptive behavioral consequences (Pascual-Leone et al., 2005). It has been shown that enriched environments and physical activities can increase the growth rate of new neurons and their maintenance in adults (Kempermann et al., 1997; Gage, 2002). However, it is still not quite understood how human brain plasticity happens throughout the life cycle (Johansen-Berg and Duzel, 2016; Walhovd et al., 2016). For example, aging is related to a gradual loss of function in multiple systems, such as the systems underlying sensation, cognition (Kramer et al., 2004), memory (de Lange et al., 2017), motor control, and emotion (Mahncke et al., 2006). In animal models, research indicates that age limits the ability to adapt to changes (Wagner et al., 2000). In contrast, studies have also suggested that older people can quickly learn new skills through training and use them completely unsupervised most of the time (Mahncke et al., 2006; Boyke et al., 2008). The accumulation of age-related brain plasticity will inevitably lead to a growing mismatch between the functional capabilities of older people and what their environment requires of them, as structural plasticity is thought to occur only when demand exceeds capacity (Lövdén et al., 2010) or when there is a considerable change in environment (de Lange et al., 2017). Moreover, an interesting longitudinal study by Brouwer et al. (2017) estimated the heritability of subcortical and global brain volume changes in five sets of twins from geologically different locations and at different stages of life, revealing genetic variants specific to brain plasticity.

Neuroplasticity responds dynamically to environmental changes, and although it is usually beneficial and adaptive, some brain plasticity can be maladaptive in some situations. This paper focuses on the environmental factor of occupation, which subtly and continuously influences the brain through various other long-term and complex factors (e.g., repetition, skilled training, and social relationship) (Falk and Bassett, 2017). External factors act as modulators or inducers of human behaviors that are based on intrinsic structures and activities of the brain. Moreover, according to research findings on neuroplasticity, occupational neuroplasticity has multiple elements, including education, lifestyle, socioeconomic status, social relationships, sustained professional training, and experience. The role of occupational neuroplasticity in the human brain over the life span is not well-understood. Thus, in the following sections, we will fully explore and conclusively infer the neurological mechanisms related to occupational neuroplasticity through a meta-analysis of many published studies. Additionally, the rationality of the neurological findings, the relationship between occupational neuroplasticity and occupational science, and the limitations and future research will be discussed.

\section{OCCUPATIONAL NEUROPLASTICITY}

Professional work styles, activities, abilities, skills, and credentials required by occupations may affect life span development of the brain and the according occupational patterns possibly associated with brain health (Habeck et al., 2019). Studies of occupational neuroplasticity at multiple levels have demonstrated that neural changes can be determined by the significance and structure of the eliciting stimulus $O n$ the one hand, neural and behavioral changes attributed to occupational neuroplasticity can happen in several months and extend to the whole lifetime. On the other hand, the stimuli's cortical representations are shaped by occupational experiences continuously on both size and temporal organization. Thus, two related questions come to the forefront: how does the brain shape occupational brain networks, and how does occupation-related connectivity shape the brain? It is difficult to study such effects, but some expert professionals, e.g., athletes, taxi drivers, musicians, dancers, simultaneous interpreters, acupuncturists, seafarers, mathematicians, pilots, and creative writers, represent ideal models in which we can investigate the potential neuroplastic changes in the brain driven by occupations. We will first review the brain changes that characterize the professionals in certain careers, followed by a quantitative meta-analysis of occupational neuroplasticity.

\section{Occupational Neuroplasticity in Athletes}

The brain of athletes provides a suitable model to study neuroplasticity, as they practice throughout their careers, usually beginning early in childhood. A recent study using voxel-based morphometry (VBM) (Ashburner and Friston, 2000) identified clusters in the right cerebellum that had higher gray matter (GM) concentration (GMC) values in experienced badminton players than in novices (Di et al., 2012). Also, this study found a greater cerebellar amplitude of low-frequency fluctuation (ALFF) in the athlete than in the control group, while the ALFF of a cluster in the left superior parietal lobe [Brodmann area (BA)7/BA19] was greater in the control group than in the athletes (Di et al., 2012). Furthermore, a study showed that GM density in the precentral gyrus and left thalamus significantly increased compared with control subjects (Wei et al., 2009). In a comparative study on brain anatomy, cortical thickness of the right parahippocampal gyrus, the right orbitofrontal cortex, and the left superior temporal sulcus was significantly increased in drivers as compared to controls (Sowell et al., 2004; Shaw et al., 2007; Jiang et al., 2009; Wei et al., 2011). Furthermore, there was a significant positive correlation between the number of years of training or the number of years of driving experience and the mean cortical thickness of the right parahippocampal gyrus, which may indicate the impact of long-term professional experience on the structure of the divers' brains (Wei et al., 2011).

Moreover, a study by Kim et al. (2011) has shown that expert archers, as compared with non-archer subjects, showed increased activation in the parahippocampal gyrus, retrosplenial cortex, and cingulate cortex, which are key brain areas of episodic memory and theory of mind-related neural networks. Through fractional anisotropy (FA) analysis of fiber tracts and VBM analyses of white matter (WM) and GM volumes, Jäncke et al. (2009) demonstrated that as compared to non-golfers or less skilled golfers with a handicap from 15 to 36, golfers with a handicap from 1 to 14 and professional golfers have larger GM volumes in the frontoparietal network.

In addition, a variety of functional reorganizations on decision making and body-related processing of the brains of expert basketball players were demonstrated by Abreu et al. (2012), 
including increased activities in the extrastriate body area during prediction, due to expert observation of action kinematics, and in the right anterior insular cortex and the bilateral inferior frontal gyrus (IFG) when making errors due to awareness of their own errors, while increased activity in the posterior insular cortex during correct action prediction may suggest the importance of body awareness on performance monitoring. Taking worldclass athletes (i.e., elite, Olympic, and internationally ranked swimmers) as an example, Huang et al. (2017) found that thalamo-sensorimotor connectivity was significantly correlated with the swimmers' motor performance excellence based on the seed-based functional connectivity (FC) analysis of resting-state functional magnetic resonance imaging data.

\section{Occupational Neuroplasticity in Taxi Drivers}

Extensive navigation experience-related structural and functional changes in the human brain were examined by comparing licensed taxi drivers and controls who were not taxi drivers (Maguire et al., 2003; Woollett et al., 2009; Wang et al., 2015; Shen et al., 2016; Peng et al., 2018). Compared to the controls, taxi drivers showed significantly increased posterior hippocampus volume positively correlated with length of employment, but decreased anterior hippocampus volume negatively correlated with length of employment (Maguire et al., 2000), suggesting local expansion of posterior hippocampus, where the environment was spatially represented, according to navigation experience. Furthermore, in terms of FC changes related to taxi drivers, Wang et al. found that in comparison to the non-drivers, the drivers had reduced intrinsic activity within the visual network and reduced FC between the sensory resting-state networks (RSNs), i.e., the primary and extrastriate visual and sensorimotor ones; additionally, the strength of the FC between the left frontoparietal and primary visual RSNs is positively correlated with length of employment (Wang et al., 2015). Regarding the functional basis of long-term navigation skill, Peng et al. (2018) investigated the role of the entorhinal cortex (EC) and found that taxi drivers had significantly reduced FC between the left anterior-lateral EC and the right anterior cingulate cortex, right angular gyrus, and bilateral precuneus and between the right posterior-medial EC and the left inferior temporal gyrus. Additionally, from the perspective of dynamical connectivity, Shen et al. (2016) found that the vigilance network of taxi drivers showed decreased amplitude of FC fluctuations, with the amplitude being negatively correlated with length of employment.

\section{Occupational Neuroplasticity in Musicians, Dancers, and Opera Performers}

The VBM analysis of Gaser and Schlaug (2003) showed differences in GM volume of the visuospatial, auditory, and motor regions of keyboard player musicians and non-musicians, and similar results were also found by Bermudez et al. (2008) when comparing non-musicians with amateur and professional musicians. They believed that the brains of musicians might have undergone structural changes to adapt to acquisition and rehearsal of professional skills. Furthermore, Bangert et al.study 2006 found increased activity in a distributed cortical network of professional pianists during both silent motion-related and acoustic tasks as compared to non-musicians. This network is composed of Wernicke's and Broca's areas and others.

Compared with non-dancers, female ballet dancers showed decreased GM volumes in the left supplementary, premotor, and motor cortices, as well as the putamen and superior frontal gyrus, decreased WM volumes in both internal capsules, both corticospinal tracts, the corpus callosum, and the left anterior cingulum; and reduced FA in the WM underlying bilateral premotor cortices (Hänggi, 2010). Recently, Lu et al. (2018) found that ballroom dancers, as compared to novices, demonstrated reduced ALFF in the left lingual gyrus; elevated ALFF in the bilateral IFG, bilateral precentral gyrus, left inferior temporal gyrus, left middle temporal gyrus, left middle frontal gyrus, left postcentral gyrus, right superior temporal gyrus (STG), and right middle occipital gyrus; and altered FC among parietal and temporal areas and the IFG.

Neuroplasticity of professional opera performers was studied by Zhang et al. (2018). Professional traditional Chinese Pingju performers, as compared with laymen, demonstrated increased regional homogeneity ( $\mathrm{ReHo}$ ) in the left anterior insula; decreased ReHo in the right middle occipital gyrus, bilateral calcarine, and superior occipital gyri and cuneus; and reduced ALFF in the bilateral cuneus and calcarine gyrus, indicating superior multidimensional performance on dancing, emotional representation, and face and music perception.

\section{Occupational Neuroplasticity in Simultaneous Interpreters}

Simultaneous interpretation makes heavy demands on executive control. In the study of Elmer et al. (2014), it was found that compared to the multilingual controls, the professional simultaneous interpreters had reduced GM volumes in the left supramarginal gyrus, pars opercularis, middle-anterior cingulate gyrus, and bilateral middle insula and pars triangularis, consistent with previous results of negative correlations between length of interpreting experience and GM volume in the bilateral caudate nucleus, left pars triangularis, and right middle-anterior cingulate gyrus and pars opercularis (Ahrens et al., 2010; Elmer et al., 2010). Hervais-Adelman et al. (2014, 2015) compared brain responses observed at both the beginning and the end of a professional training program in simultaneous interpretation, and their results suggested the importance of the caudate nucleus as a central node in networks related to this occupation.

\section{Occupational Neuroplasticity in Acupuncturists}

In a study of brain structural changes among professional acupuncturists, Dong et al. (2013) revealed significantly larger GM volumes in acupuncturists than in non-acupuncturists in the bilateral ventral medial prefrontal cortex/ventral anterior cingulate cortex (VMPFC/vACC), the right cerebellar lobule V/VI, and the left primary somatosensory cortex (SI). Further studies revealed a positive correlation between the duration 
of acupuncture practice and GM volumes of the left SI and cerebellar V/VI (Dong et al., 2014, 2015). Moreover, in terms of functional alterations, Cheng et al. (2007) were the first to demonstrate that when observing an animation of acupuncture practice, in comparison to naive participants, acupuncturists have increased activation of the temporoparietal junction and the medial and superior prefrontal cortices, which are involved in emotion regulation. Dong et al. (2014) investigated the ReHo alteration of acupuncturists and found that the acupuncturists showed increased ReHo in the left SI, the left primary motor cortex (MI), and the left VMPFC/orbitofrontal cortex. Additionally, Dong et al. (2015) explored the ALFF indicators related to the brain activity of acupuncturists, which demonstrated increased ALFF for acupuncturists in the contralateral hand representation area of the SI and the left VMPFC.

\section{Occupational Neuroplasticity in Seafarers}

Regarding professional seafarers, a resting-state fMRI study by Wang et al. (2017) demonstrated for the first time that seafarers have a distinct atomic connectome pattern (ACP), i.e., ACP14, supporting their vocational requirements; this pattern consists of four specific subnetworks: the visual, auditory, vestibular, and executive control networks. Moreover, Wang et al. (2018) found that the entropy of the STG and orbital-frontal gyrus was significantly higher in seafarers than in non-seafarers, while the cerebellar entropy of the seafarers was lower than that of the controls. The above results imply that seafarers have a more specialized cerebellum and lower capacity for auditory information processing and emotional control than non-seafarers have (Zeng et al., 2016; Wang et al., 2017).

\section{Occupational Neuroplasticity in Mathematicians}

Mathematicians, as members of a highly specialized occupation, require extensive professional training over many years. Aydin et al. (2007) found that mathematicians showed significantly increased cortical GM density in the bilateral inferior parietal and left inferior frontal lobules compared with control subjects. Furthermore, Amalric and Dehaene (2016) found that professional mathematicians showed a reproducibly activated set of bilateral ventrolateral temporal, intraparietal, and frontal regions when facing mathematical statements in geometry, topology, analysis, or algebra. Additionally, Popescu et al. (2019) investigated the structural brain correlates of mathematical expertise, and they found that the mathematicians had increased GM density in the right superior parietal region and decreased GM density in the left IFG and the right intraparietal sulcus. Interestingly, abacus experts (Tanaka et al., 2002) have shown increased brain functional activity in certain cortical areas such as the bilateral superior parietal lobule and superior frontal sulcus that were connected to visuospatial working memory, suggesting that abacus experts utilize visuospatial representations for digital memory.

\section{Occupational Neuroplasticity in Professional Chess Players}

The professional chess player group with long-term training and outstanding logical thinking also provides a suitable model to study the high-level cognition related to cognitive expertise. According to an fMRI study on visual system responding to chess (Krawczyk et al., 2011), it was found that the right temporal cortex, orbitofrontal cortex, and posterior cingulate were more active in chess experts than in novices when observing chess as compared to scrambled chess. Further, the Chinese chess player within resting state showed increased FC between the hippocampus, thalamus, basal ganglia, and parietal/temporal region, denoting the expertise influence on learning-and memory-associated intrinsic connectivity networks (Duan et al., 2014), while in the chess problem-solving task, a broader task-induced deactivation of the default mode network (DMN) in experts was found (Duan et al., 2012). Moreover, a functional network hub analysis was recently performed on the intrinsic FC related to chess experts, which revealed increased FC strength in the right posterior fusiform gyrus as well as its connection to the visuospatial attention and motor networks, in experts over novices (Song et al., 2019). Benefiting from diffusion magnetic resonance imaging (dMRI) studies, structural (WM) differences have been reported in which the bilateral superior longitudinal fasciculus (SLF), inferior longitudinal fasciculus (ILF), and inferior fronto-occipital fasciculus (IFOF) were directly correlated with duration of training in chess experts (Mayeli et al., 2018). Consistently, Feng et al. (2020) detected significant differences in the thalamo-frontal tracts and left SLF. Additionally, a multimodal MRI dataset (29 Chinese chess players and 29 age-matched novices) was released by $\mathrm{Li}$ et al. (2015), which could help researchers to further explore underlying neural mechanisms related to chess expertise.

\section{Neuroplasticity Related to Other Occupations}

Ahamed et al. (2014) investigated the brain structural changes of glider pilots in contrast to non-pilots using VBM analysis, which revealed significant increases in GM density in glider pilots in brain areas associated with motor and cognitive processes when piloting a glider, including the left supplementary eye field, anterior cingulate cortex, and ventral premotor cortex. Additionally, Neumann et al. (2018) explored the structural changes in creative writers' brains and found that expert writers had increased GM volumes in the right middle frontal and superior frontal gyri (BA9 and BA10) as well as the left middle frontal gyrus (BA9, BA10, and BA46), left posterior cerebellum, and bilateral medial dorsal nuclei of the thalamus. Interestingly, studies (Kawabata and Zeki, 2004; Vartanian and Goel, 2004) have shown that expertise influences aesthetic judgments, and architects with aesthetic expertise (Kirk et al., 2009) showed differential activation of the bilateral subcallosal cingulate gyrus and medial orbitofrontal cortex compared to non-architects during this judgment. Furthermore, sommeliers (wine experts) (Pazart et al., 2014) also showed activation in the temporal pole and hippocampal and parahippocampal formations when 
tasting wine; Sreenivasan et al. (2017) have explored the influence of expertise on causal connectivity and topological property related to master sommeliers during different olfactory and non-olfactory tasks, where in sommeliers, a significantly greater connectivity involving the precuneus, caudate, putamen, and several frontal and temporal regions was observed and a significantly higher small-world topology was identified.

In summary, we can see that the occupational factors are indeed able to shape changes in brain structure and function, which are beneficial results of strong occupational neuroplasticity in the human brain.

\section{META-ANALYSIS}

Meta-analysis combines a corpus of related studies and extracts concordant findings from them. Activation likelihood estimation (ALE), developed by Turkeltaub et al. (2002), is a most widely used quantitative approach to meta-analysis of neuroimaging studies, utilizing the fact that most functional and structural neuroimaging studies report $3-\mathrm{D}(x, y, z)$ coordinates of activity foci in standardized stereotactic space (Laird et al., 2005, 2009; Bzdok et al., 2012; Turkeltaub et al., 2012). For example, Caspers et al. (2010) summarized and amended the previous knowledge of human brain networks, indicating a bilateral network within the parietal, temporal-occipital, and frontal premotor cortices by conjunction meta-analyses of neuroimaging studies on action observation and imitation. Fuelscher et al. (2018) first took advantage of ALE analysis to detect reliable neural correlates of developmental coordination disorder symptoms. Largely based on the recent growth in neuroimaging studies, there are a growing number of suggestions on how best to conduct a meta-analysis (Peyron et al., 2000; Wright et al., 2000; Müller et al., 2018), which have contributed to an integrated view of the brain, and previous studies have provided several methods to analyze enormous functional and structural datasets. In this study, we will we will take advantage of meta-analysis techniques to perform a well-structured metaanalysis of functional and structural neuroimaging studies related to occupational neuroplasticity, respectively.

\section{Paper Selection and Screening}

In this study, we followed the Preferred Reporting Items for Systematic Reviews and Meta-Analysis (PRISMA) guidelines

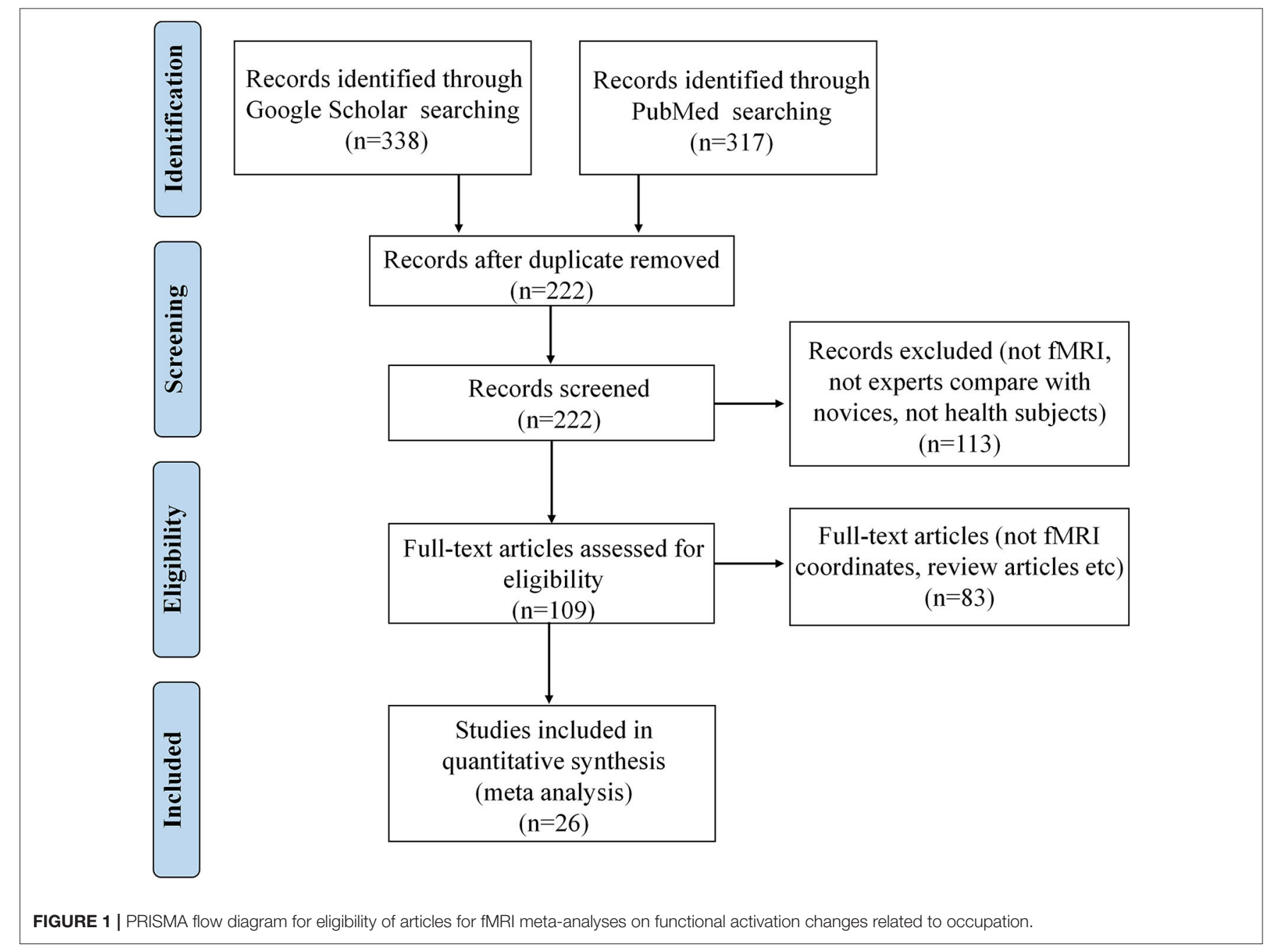


(Moher et al., 2010), and we searched PubMed (http://www. ncbi.nlm.nih.gov/pubmed/) and Google Scholar (https://scholar. google.com) for papers. The keywords included three parts: the research field (e.g., "expert," "expertise," "professional," or "specialist"), the focus on brain functions (e.g., "brain" or "cortical"), and the neuroimaging technique (e.g., "fMRI", "functional MRI," or "functional magnetic resonance imaging"). Literature published from 1999 to 2019 was included in this meta-analysis.

In terms of meta-analysis of functional neuroimaging studies related to occupational neuroplasticity, only studies that analyzed local changes in the brain cortex based on fMRI were included in our functional meta-analysis. In total, 26 studies were included, while hundreds of studies had to be excluded for the following reasons:

(1) The studies did not compare experts with novices.

(2) The studies did not report on brain activation as an experimental result.

(3) The studies contained fewer than three stereotactic coordinates or did not report coordinates at all.

The corresponding PRISMA flowchart for eligibility of articles for fMRI meta-analyses on functional activation changes was presented in Figure 1, and 26 studies were ultimately included (for details, see Table 1).

In terms of meta-analysis of structural neuroimaging studies related to occupational neuroplasticity, we also conducted a comprehensive search for structural MRI morphometric studies on PubMed and Google Scholar (search strings: "player + morphometry," "player + brain + mri," "player + brain + VBM," "expert + morphometry," "expert + brain + mri," and "expert + brain + VBM"). Literature published from 1999 to 2018 was included.

Only studies that analyzed local changes in GM based on structural MRI were included in our structural meta-analysis. In total, 17 studies were included, and some studies were excluded due to the following reasons:

(1) The studies did not compare experts with novices.

(2) The studies reported only volumetric data.

(3) The studies contained fewer than three stereotactic coordinates or did not report coordinates at all.

The corresponding PRISMA flowchart for eligibility of articles for MRI meta-analyses on volume changes was presented in Figure 2, and 17 studies were finally included (for details, see Table 2).

\section{Analytical Procedure}

GingerALE 2.3.6 (UT Health Science Center Research Imaging Institute, San Antonio, TX) was used as an implementation of ALE quantitative meta-analysis of neuroimaging studies with a random-effects model, combined with cluster-level inference with a false discovery rate (FDR) (Genovese et al., 2002) of $q=0.05$.

Coordinates of peak voxels in those studies originally reported in the Talairach system were converted to the Montreal Neurological Institute (MNI) system using the WFU PickAtlas
TABLE 1 | Original fMRI studies included in the functional meta-analysis.

\begin{tabular}{|c|c|c|c|c|}
\hline References & Expertise & $\begin{array}{l}\text { Expert } \\
(N)\end{array}$ & $\begin{array}{l}\text { Novice } \\
\text { (N) }\end{array}$ & Task or Rest \\
\hline Abreu et al. (2012) & $\begin{array}{l}\text { Basketball } \\
\text { players }\end{array}$ & 16 & 16 & Motor prediction \\
\hline Balser et al. (2014) & Tennis players & 16 & 16 & Motor prediction \\
\hline Bangert et al. (2006) & Pianists & 7 & 7 & Auditory and motor \\
\hline Baumann et al. (2007) & Pianists & 7 & 7 & Motor execution \\
\hline $\begin{array}{l}\text { Berkowitz and Ansari } \\
\text { (2010) }\end{array}$ & Pianists & 13 & 15 & Motor execution \\
\hline Bishop et al. (2013) & Soccer players & 14 & 11 & Motor prediction \\
\hline Chang et al. (2011) & Archery & 18 & 18 & Motor imagery \\
\hline Di et al. (2012) & $\begin{array}{l}\text { Badminton } \\
\text { players }\end{array}$ & 20 & 18 & Rest \\
\hline Duan et al. (2012) & $\begin{array}{l}\text { Chinese chess } \\
\text { players }\end{array}$ & 15 & 15 & $\begin{array}{l}\text { Rest/problem-solving } \\
\text { task }\end{array}$ \\
\hline Groussard et al. (2010) & Musicians & 20 & 20 & $\begin{array}{l}\text { Musical semantic } \\
\text { memory }\end{array}$ \\
\hline $\begin{array}{l}\text { Harris and de Jong } \\
\text { (2014) }\end{array}$ & $\begin{array}{l}\text { Keyboard } \\
\text { players }\end{array}$ & 12 & 12 & Motor imagery \\
\hline Haslinger et al. (2004) & Pianists & 12 & 12 & Motor execution \\
\hline Kim et al. (2011) & Archery & 20 & 21 & Motor observation \\
\hline Koeneke et al. (2004) & $\begin{array}{l}\text { Keyboard } \\
\text { players }\end{array}$ & 7 & 7 & Motor execution \\
\hline Krawczyk et al. (2011) & Chess players & 6 & 6 & Face processing \\
\hline $\begin{array}{l}\text { Lee and Noppeney } \\
\text { (2011) }\end{array}$ & Pianists & 18 & 19 & Music listening \\
\hline Lotze et al. (2003) & Violinists & 8 & 8 & $\begin{array}{l}\text { Motor execution and } \\
\text { imagination }\end{array}$ \\
\hline Luo et al. (2012) & Musicians & 15 & 15 & $\begin{array}{l}\text { Motor and } \\
\text { multi-sensory }\end{array}$ \\
\hline Pilgramm et al. (2010) & Dancers & 18 & 18 & Motor observation \\
\hline Seo et al. (2012) & Archery & 20 & 23 & Visuospatial memory \\
\hline Song et al. (2019) & Chess players & 28 & 27 & Rest \\
\hline Tomasino et al. (2013) & Volleyball players & 10 & 10 & $\begin{array}{l}\text { Motor language } \\
\text { processing }\end{array}$ \\
\hline Wang et al. (2018) & Seafarers & 20 & 20 & Rest \\
\hline Wright et al. (2011) & $\begin{array}{l}\text { Badminton } \\
\text { players }\end{array}$ & 8 & 8 & Motor prediction \\
\hline Wright et al. (2013) & Soccer players & 17 & 17 & Motor observation \\
\hline Xu et al. (2016) & $\begin{array}{l}\text { Badminton } \\
\text { players }\end{array}$ & 16 & 18 & Motor prediction \\
\hline
\end{tabular}

software (Maldjian et al., 2003). Multi-Image Analysis GUI ("Mango," UT Health Science Center Research Imaging Institute) was used to display our ALE meta-analytic findings (see Results section below).

\section{RESULTS}

\section{Brain Activation Changes Related to Occupation}

\section{Functional Contrast: Experts > Novices}

The meta-analysis of functional activation changes in this contrast included 26 studies with 365 foci. The results indicated 


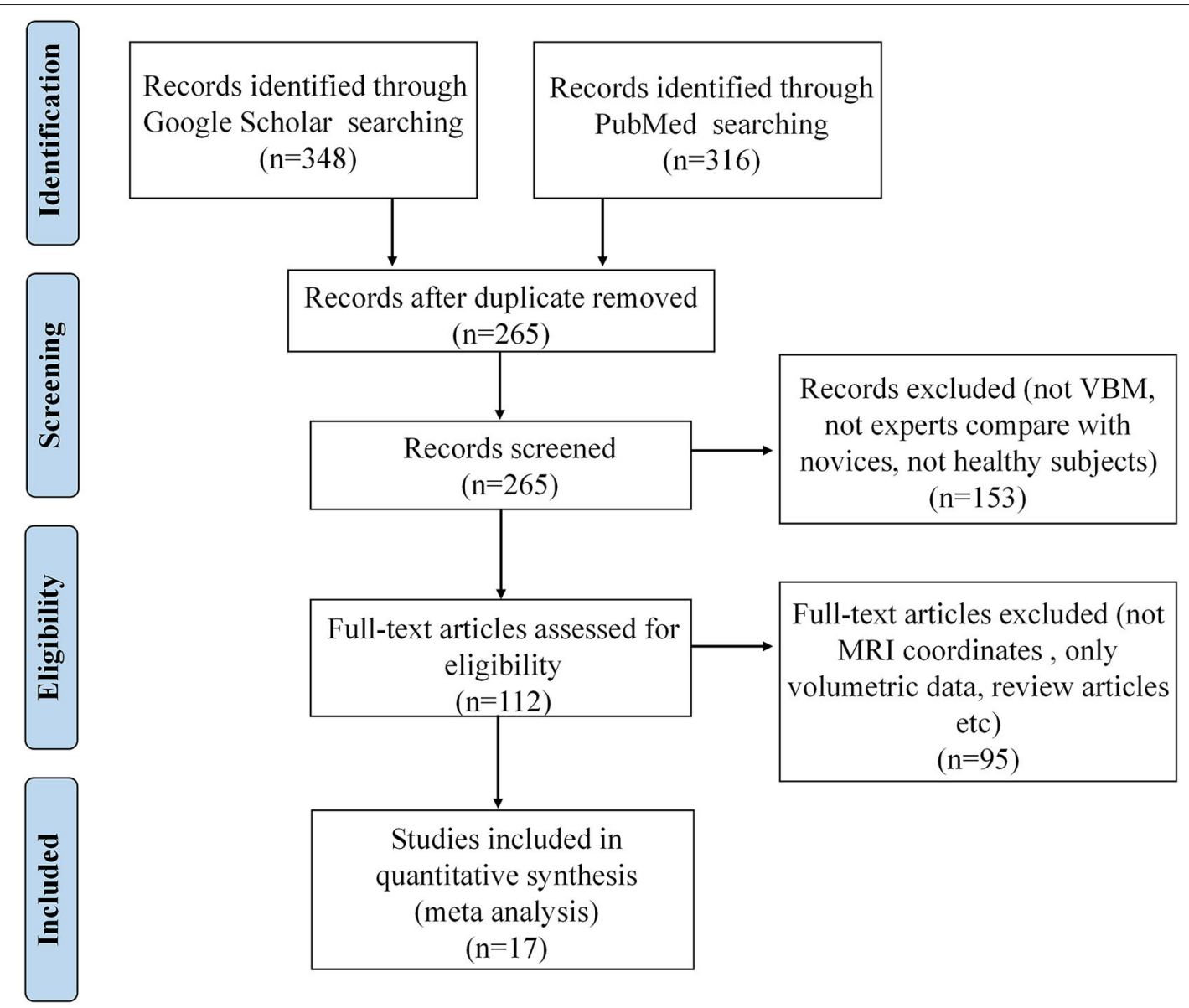

FIGURE 2 | PRISMA flow diagram for eligibility of articles for MRI meta-analyses on structural volume changes related to occupation.

six significant clusters located in BA6 and BA9, which are shown in Table 3 and Figures 3, 4.

\section{Structural Volume Changes Related to Occupation}

Structural Contrast: Experts > Novices

The meta-analysis of structural changes in this contrast included 17 studies with 101 foci. The results indicated three significant clusters located in the right putamen, the left STG (BA22), and the right STG (BA22), which are shown in Table 4 and Figure 5.

\section{DISCUSSION}

\section{Occupational Science and Occupational Neuroplasticity}

Occupational science is the study of human occupation, which has two elements, i.e., the human and the occupation (Hocking, 2009). The central concept within occupational science is occupation itself, which is mainly characterized by diversity. A conceptual research framework with respect to occupational science consists of the essential elements of occupation and occupational processes as well as the relationship between occupation and other phenomena (Hocking, 2000). In terms of the essential elements of occupation, the focus of research is to identify the nature, structure, substrates, and features of occupation. With respect to occupational processes, the focus of research is to investigate the process, outcomes, features, and subjective experience of occupational performance. Considering the relationship between occupation and other phenomena, the focus of research is to explain how occupation relates to identity, health, human development, quality of life, and social structures and policies (Hocking, 2000). As seen from the above, occupation and the related occupational science are very complex, diverse, and comprehensive.

The concept of "occupational neuroplasticity," as introduced in this paper, can be treated as a potential branch of occupational science that focuses on disclosing the relationship between the brain's plasticity (functional neuroplasticity, structural neuroplasticity, etc.) and long-term occupational training or experience. Due to the diversity and complexity of occupation, occupational neuroplasticity shows the heterogeneity among many different types of occupation, with a complex intrinsic mechanism and an ongoing process of comprehensive change related to occupational development and other factors such as health status, mental illness, and neuropsychiatric or neurological 
TABLE 2 | Original MRI studies included in the structural meta-analysis.

\begin{tabular}{llcc}
\hline References & Expertise & $\begin{array}{c}\text { Expert } \\
\text { (N) }\end{array}$ & $\begin{array}{c}\text { Novice } \\
\text { (N) }\end{array}$ \\
\hline Ahamed et al. (2014) & Glider Pilots & 15 & 15 \\
Bermudez et al. (2008) & Musicians & 71 & 64 \\
Di et al. (2012) & Badminton Players & 20 & 18 \\
Fauvel et al. (2014) & Musicians & 16 & 17 \\
Groussard et al. (2014) & Musicians & 22 & 22 \\
Hänggi (2010) & Ballet Dancers & 10 & 10 \\
Hernández et al. (2016) & Sahaja Yoga Meditators & 23 & 23 \\
Hu et al. (2018) & Track Athletes/Badminton & $13 / 13$ & 16 \\
& Players & & \\
Huang et al. (2015) & Gymnasts & 13 & 13 \\
Hüfner et al. (2011) & Dancers \& Slackliners & 21 & 20 \\
Jäncke et al. (2009) & Golfers & 20 & 20 \\
James et al. (2014) & Musicians & 18 & 19 \\
Kleber et al. (2016) & Opera Singers & 27 & 28 \\
Li et al. (2011) & Musicians & 15 & 15 \\
Li et al. (2018) & Musicians/Dancers & $20 / 18$ & 25 \\
Tan et al. (2017) & Basketball Players & 21 & 21 \\
Vaquero et al. (2016) & Pianists & 36 & 17 \\
\hline
\end{tabular}

disease. Specifically, as mentioned before, occupational neuroplasticity is associated with the process of acquiring professional skills through sustained performance. This process comprises multiple elements, i.e., professional acquisition, interpersonal relationships, social reward and competition systems, consistent work environments, and possible genetic factors; these numerous elements make the process very complex and comprehensive. Taking environmental factors as an example, toxins, drugs, genetic mutations, nutritional disturbances, and environmental sensory and behavioral conditions all affect neuronal network formation at multiple levels in the developing brain (Fulton, 1935). This might provide new perspectives into the mechanisms of occupational neuroplasticity, where various occupational environments cause both benefits and drawbacks and are closely related to mental health, aging, mental disorders, neurological diseases, neurodegenerative diseases, etc. Thus, the exploration of occupational neuroplasticity can not only help uncover the commonly intrinsic neurophysiological mechanisms shaped by occupation (the main focus of this paper) but also accumulate knowledge and data on mental health promotion, prevention, and intervention in occupational therapy (Haglund and Henriksson, 2003; Arbesman et al., 2013; Read et al., 2018).

\section{Functional Neuroplasticity Driven by Occupation}

Through the meta-analysis of functional neuroimaging studies related to occupation, we found that the cortical regions BA6 and BA9 were significantly related to the occupational experience training, providing some functional evidences of occupational neuroplasticity, as we discussed below.

We will begin by discussing BA6, a traditional "motor" area that many studies suggest to be active during higher motor control involving a variety of cognitive operations (Fulton, 1935;
TABLE 3 | Strengthened activation results in experts compared with novices in functional neuroimaging meta-analysis.

\begin{tabular}{lcccll}
\hline Volume $\left(\mathbf{m m}^{\mathbf{3}}\right)$ & $\boldsymbol{x}$ & $\boldsymbol{y}$ & $\boldsymbol{z}$ & Label & Brodmann area \\
\hline 707 & -54 & 4 & 32 & L Precentral Gyrus & BA6 \\
160 & 0 & -20 & 56 & L Paracentral Lobule & BA6 \\
641 & 4 & -4 & 68 & R Medial Frontal Gyrus & BA6 \\
210 & -38 & 16 & 30 & L Precentral Gyrus & BA9 \\
833 & -22 & 0 & 62 & L Middle Frontal Gyrus & BA6 \\
572 & -44 & 14 & 46 & L Middle Frontal Gyrus & BA6 \\
\hline
\end{tabular}

Wise, 1985; Freund, 1990). Some neuroanatomical evidence suggests that although the caudal region of BA6 is closely related to the primary motor cortex and produces a large number of corticospinal projections, the rostral region of BA6 is closely related to the prefrontal cortex rather than the primary motor cortex, sending no direct projections to the latter (Barbas and Pandya, 1987; Luppino et al., 1993; Lu et al., 1994). However, research results on this topic are inconsistent, and the structural and functional roles of BA6 in cognition and in motor control are unclear (Picard and Strick, 2001; Schubotz and von Cramon, 2003). Functional activation in BA6 during cognitive tasks is often interpreted as accompanying potential motor processes, such as preparation for button pressing or eye movement; therefore, it is not out of the question for the functional relevance of BA6 activation in cognition (Courtney et al., 1998). Furthermore, BA6 has modality-specific cognitive functions (Tanaka et al., 2005), and there is growing evidence that some classically designated "motor" regions also take functions in non-motor cognitive processes (Ito, 1993; Leiner et al., 1993; Middleton and Strick, 1994; Tanaka et al., 2005). Additionally, convergent pieces of evidence from neuroimaging studies suggest a unique role for part of BA6, i.e., the medial frontal cortex, which is closely related to social cognitive processing (Amodio and Frith, 2006). Considering the complexity and diversity of occupation and the results found in this study, long-term experience with occupational training potentially causes the significant alternations in motor control, non-motor cognitive function, and social cognitive processing among experts in contrast to novices.

The region of BA9 focused on in this paper, i.e., the IFG, is considered to be related to general cognitive function, which is involved in working memory (Zhang et al., 2003; Schulze et al., 2011), cognitive control of memory (Badre and Wagner, 2007), selection or inhibition of other self-control functions (Goghari and MacDonald, 2009), cognitive flexibility (Ghahremani et al., 2009), speech and language processing (Greenlee et al., 2007), inhibitory processes (Sharot et al., 2012), etc. This suggests that long-term occupational training experience is a general, comprehensive, ongoing training process that is associated with many factors in occupational training. Furthermore, the strengthened effects in experts in the right IFG of BA9 and the left precentral gyrus (BA6), part of the mirror neuron system (Rizzolatti et al., 2014), suggest that these areas become more involved in imitating other people's movements to understand 


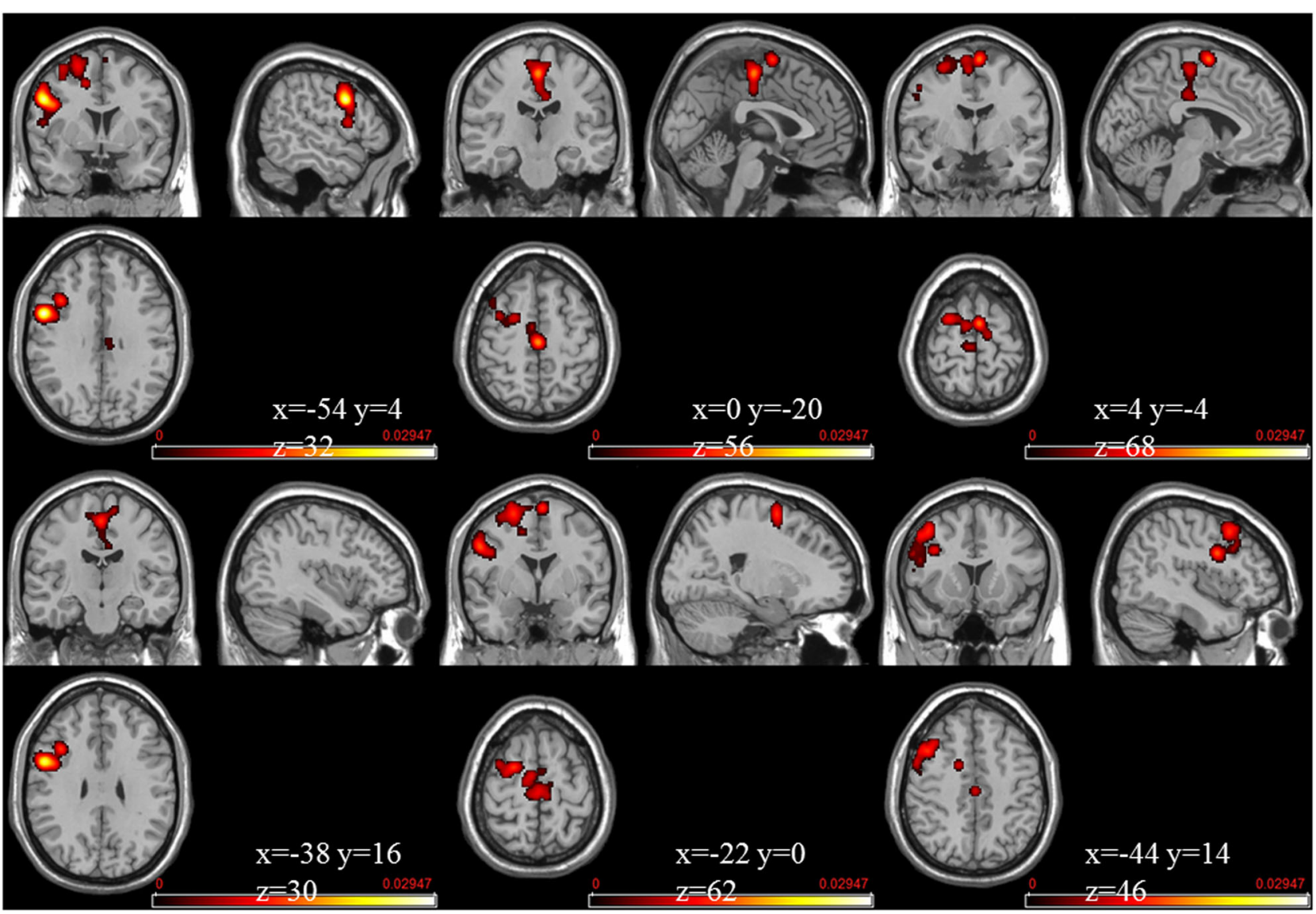

FIGURE 3 | Strengthened activation estimated by ALE with cluster-level correction (FDR of $q=0.05)$ in experts compared with novices.

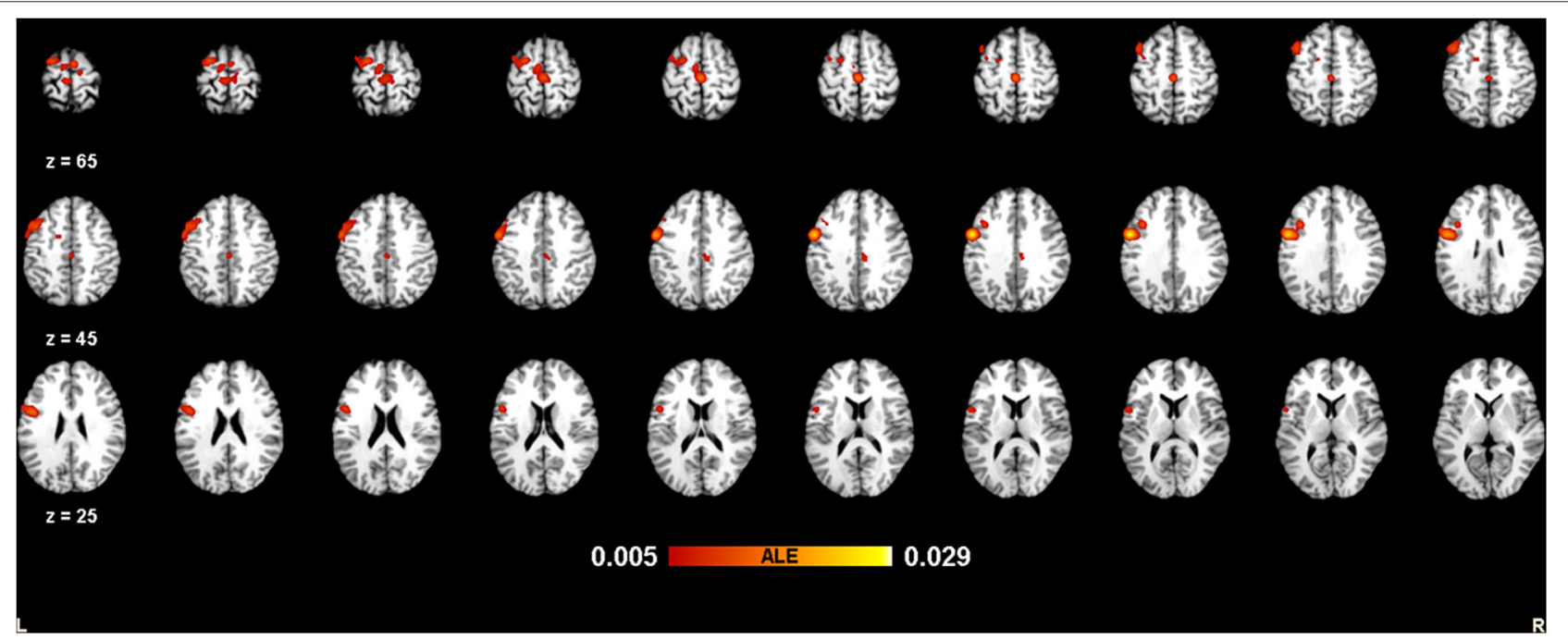

FIGURE 4 | Strengthened activation estimated by ALE with cluster-level correction (FDR of $q=0.05$ ) in experts compared with novices, shown in a multislice view.

the corresponding emotions and intentions after long-term occupational training (Molnar-Szakacs and Overy, 2006) in one's career. Indeed, according to previous studies, experts have a larger left frontal area than controls (Sluming et al., 2002), and GM density increases with musical expertise (Gaser and Schlaug, 2003; James et al., 2014). 
According to the aforementioned discussion, due to the complexity, diversity, and comprehensiveness of occupation, the long-term training required by one's occupation may create specifically intrinsic representations to match the demands of one's own career, involving functions such as sensation, movement, non-motor cognition, social cognitive processing, working memory, and speech and language processing.

\section{Structural Neuroplasticity Driven by Occupation}

Many studies have suggested that structural neuroplasticity may evolve in all directions under influence modulation of certain skill learning and expertise (Chang, 2014). In this present study, as compared to novices, experts showed greater GM volume in the right putamen, classically associated with motor control and more recently with memory-related processes, reinforcement learning, and implicit sequence learning (Vaquero et al., 2016). This phenomenon may imply that the functions of movement, learning, and memory are very important in the occupational

TABLE 4 | Volume increase results in experts compared with novices in structural neuroimaging meta-analysis.

\begin{tabular}{lcccll}
\hline Volume $\left(\mathbf{m m}^{\mathbf{3}}\right)$ & $\boldsymbol{x}$ & $\boldsymbol{y}$ & $\boldsymbol{z}$ & Label & Brodmann area \\
\hline 1984 & -52 & -8 & 2 & L Superior Temporal Gyrus & BA22 \\
1200 & 28 & 12 & -10 & R Putamen & - \\
80 & 56 & -16 & 4 & R Superior Temporal Gyrus & BA22
\end{tabular}

training process. Another cortical area with a larger GM volume in experts than in novices was the STG (BA22), which contains the primary auditory area (Wernicke's area) and shows a key role in language processing. The left STG region is involved in the understanding and generation of individual words, while the right STG is involved in discriminating sound intensity and pitch. Additionally, the left auditory cortex may process rapid changes in temporal properties more precisely than the right auditory cortex (Schneider et al., 2002; Zatorre, 2013). Thus, according to the previous discussion of structural neuroplasticity, we can speculate that occupation-related training or experience possibly impacts the capacity for auditory and language processing, motor functions, learning, and memory, which play key roles in carrying out the functions of one's occupation and provide some structural evidence of occupational neuroplasticity.

\section{Target Areas for Occupational Neuroplasticity Modulation}

In this research, some common regions (see Figures 3-5) were robustly identified by meta-analysis in terms of occupational neuroplasticity across a lot of occupations, which demonstrates a common neural mechanism of occupational neuroplasticity. Further, these identified common regions in terms of structural or functional changes possibly are treated as the target ones in daily and specifically designed occupational training activities and through neuromodulation approaches, i.e., transcranial magnetic stimulation (TMS) (Pascual-Leone et al., 2002), transcranial direct current stimulation (tDCS) (Paulus, 2011), etc., which possibly improve the occupational capability. For

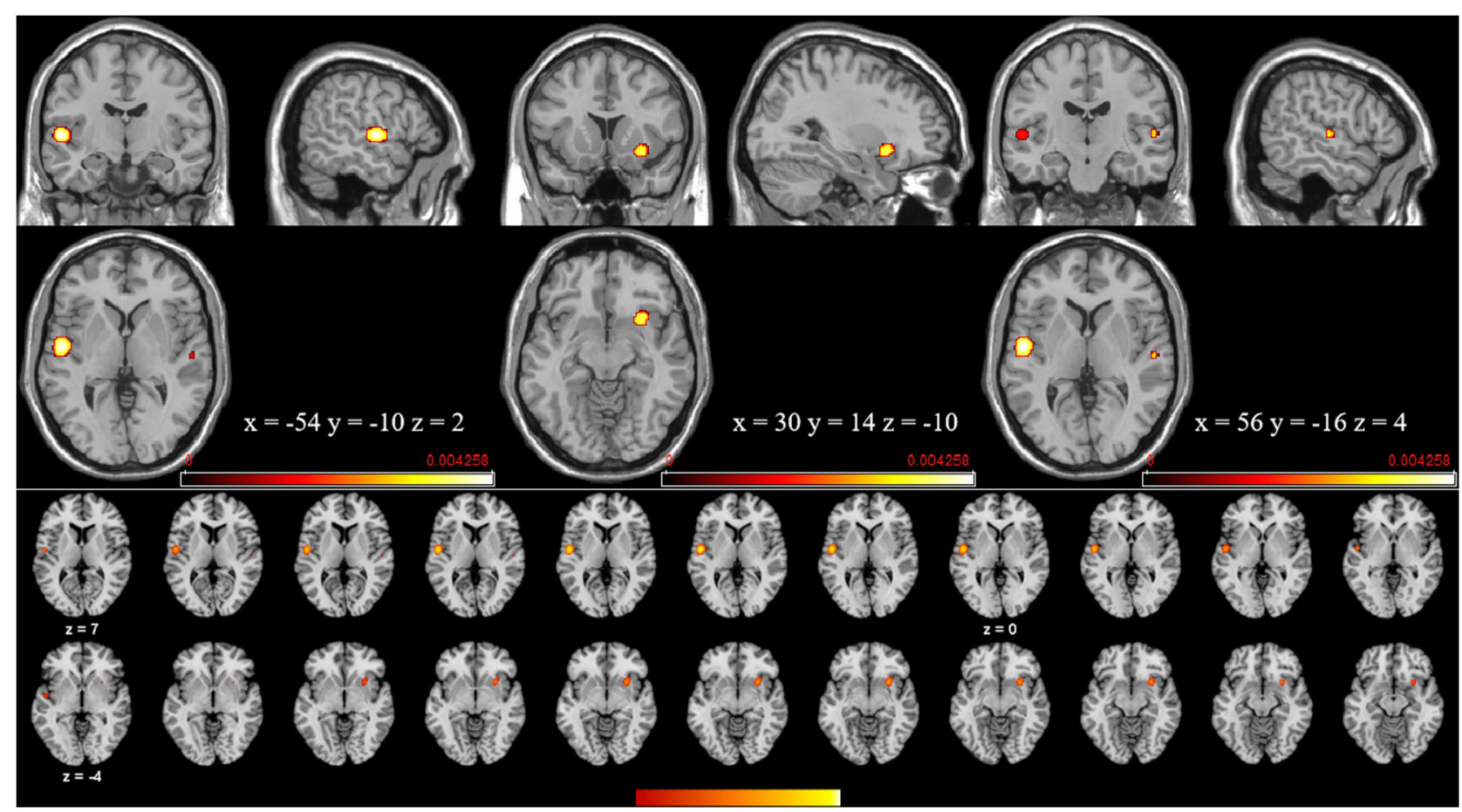

FIGURE 5 | Volume increases estimated by ALE with cluster-level correction (FDR of $q=0.05$ ) in experts compared with novices. 
example, Reis et al. (2008) reviewed many related papers and found that the aforementioned stimulation techniques could regulate memory formation and motor learning in healthy humans. Also, many researches demonstrated that the above stimulation techniques could be an effective treatment for psychiatric disorders (e.g., major depressive disorder) (Reis et al., 2008; Liu et al., 2017) and neurodegenerative dementias (Elder and Taylor, 2014). Thus, based on previous studies, the identified portions (listed in Tables 3, 4) of BA6, BA9, and BA22 are likely located as targets in neural modulation of occupational capability at the individual or group level.

\section{Inconsistency of Structural and Functional Alterations Concerning Occupation}

According to the presented meta-analysis results about the brain structure and function regarding occupation, the inconsistent alterations were observed, which may result from the following points. At the beginning, the included papers in structural and functional meta-analyses were not identical, because the results of both functional and structural alterations regarding a certain occupation were less reported simultaneously in a paper. This brought great subject diversity in the structural meta-analysis and functional meta-analysis. Further, the reported VBM results from the included papers quite depended on the resolution of T1 brain images, where magnetic field intensity had obvious main effects (Obusez et al., 2018). The recently developed quantitative MRI (qMRI) with its robustness and effectiveness could be an alternative to investigate the brain structure changes corresponding to occupation in the future (Weiskopf et al., 2013). In addition, this phenomenon likely originates from the intrinsic inconsistency between anatomical connectivity and FC (Park and Friston, 2013; Messé et al., 2014). For example, Park and Friston (2013) pointed out that the divergence of function from structure is the most intriguing property of the brain; Messé et al. (2014) also revealed that anatomical connectivity alone accounts for up to $15 \%$ of FC variance. To sum up, exploring the connection between the functional neuroplasticity and structural neuroplasticity with regard to occupation is a key part of occupational neuroplasticity in future studies.

\section{Limitations and Future Research}

The present investigation has some limitations. First, due to the heterogeneity and diversity of occupation, there are few studies on certain types of occupations, which imposed some restrictions on exploring the mechanism at the individual occupation level by means of meta-analysis. Thus, in the future, we advocate more studies on occupational neuroplasticity, which contributes to the understanding of the intrinsic mechanism of occupational neuroplasticity at the group or individual occupation level. Second, in this paper, we reported some neuroimaging results that included only the changes of GM and functional activation due to lack of WM studies; extensive research on occupational neuroplasticity could remedy this lack of data on WM. Third, the relationship between occupational neuroplasticity and the duration of occupational experience also can be further investigated. In addition, how the level of work complexity is closely related to the occupational neuroplasticity should be addressed in the future. Finally, whether occupational neuroplasticity is predominantly determined by nature (internal genes) or nurture (external experimental factors) or both remains an open question and needs to be explored carefully in future studies.

\section{CONCLUSION}

Advances in neuroimaging over the past few decades have improved our understanding on the neuroplastic changes due to skill learning, expertise, etc., in terms of occupation. Based on the meta-analysis, these plastic changes can be demonstrated clearly at structural and functional levels (Figures 3-5), suggesting the possible common neurophysiological basis of occupational neuroplasticity. In terms of functional reorganization, a common finding in experts is a set of changes mainly centered on activation of brain cortical areas BA6 (mainly including the medial frontal gyrus, middle frontal gyrus, precentral gyrus, and paracentral lobule) and BA9 (including the IFG), likely in order to satisfy occupational requirements by strengthening functions such as sensation, movement, non-motor cognition, social cognitive processing, working memory, and speech and language processing. Meanwhile, in terms of structural reorganization, structural analysis reveals that experts show increased GM volume in the cortical region BA22 (included in the STG) and in the putamen, possibly supporting occupationally necessary abilities such auditory and language processing, movement, learning, and memory. The outlined areas could be used as the likely targets for modulating occupational neuroplasticity, aiming at improving the comprehensive occupational capability and further revealed the commonly or basically underlying neural mechanism across different kinds of occupation. Moreover, we discussed the rationality of the occupation-related functional and structural differences discovered in experts compared with novices and the inconsistent alterations of structural and functional reorganization concerning occupation factor. Finally, we thoroughly reviewed pieces of evidence related to occupational neuroplasticity in many types of occupations, such as athletes, taxi drivers, musicians and dancers, simultaneous interpreters, acupuncturists, seafarers, mathematicians, pilots, and creative writers. In summary, our findings enriched the conceptualization of neuroplasticity, partly defined the neural basis of occupational neuroplasticity, and provided a new perspective for occupational science research.

\section{DATA AVAILABILITY STATEMENT}

All datasets generated and analyzed for this study are included in the article/supplementary material.

\section{AUTHOR CONTRIBUTIONS}

HW and HY analyzed the data and wrote the draft. NW conceived of the study, checked the results, and wrote the paper. YY, MX, YS, WZ, JL, and JZ discussed the study, read the manuscript, and gave feedback. CC discussed the 
study extensively, read and revised the draft, and provided commentary. All authors contributed to the article and approved the submitted version.

\section{FUNDING}

This work was supported by the National Natural Science Foundation of China (Nos. 61701318 and 61971289), Natural

\section{REFERENCES}

Abreu, A. M., Macaluso, E., Azevedo, R. T., Cesari, P., Urgesi, C., and Aglioti, S. M. (2012). Action anticipation beyond the action observation network: a functional magnetic resonance imaging study in expert basketball players. Eur. J. Neurosci. 35, 1646-1654. doi: 10.1111/j.1460-9568.2012. 08104.x

Ahamed, T., Kawanabe, M., Ishii, S., and Callan, D. E. (2014). Structural differences in gray matter between glider pilots and non-pilots. A voxel-based morphometry study. Front. Neurol. 5:248. doi: 10.3389/fneur.2014.00248

Ahrens, B., Kalderon, E., Krick, C. M., and Reith, W. (2010). fMRI for exploring simultaneous interpreting. Why Transl. Stud. Matter. 88, 237-248. doi: $10.1075 / \mathrm{btl} .88 .20 \mathrm{ahr}$

Amalric, M., and Dehaene, S. (2016). Origins of the brain networks for advanced mathematics in expert mathematicians. Proc. Natl. Acad. Sci. U.S A. 113, 4909-4917. doi: 10.1073/pnas.1603205113

Amodio, D. M., and Frith, C. D. (2006). Meeting of minds: the medial frontal cortex and social cognition. Nat. Rev. Neurosci. 7, 268-277. doi: $10.1038 / \mathrm{nrn} 1884$

Arbesman, M., Bazyk, S., and Nochajski, S. M. (2013). Systematic review of occupational therapy and mental health promotion, prevention, and intervention for children and youth. Am. J. Occup. Ther. 67, e120-e130. doi: 10.5014/ajot.2013.007039

Ashburner, J., and Friston, K. J. (2000). Voxel-based morphometry-the methods. Neuroimage 11, 805-821. doi: 10.1006/nimg.2000.0582

Aydin, K., Ucar, A., Oguz, K. K., Okur, O. O., Agayev, A., Unal, Z., et al. (2007). Increased gray matter density in the parietal cortex of mathematicians: a voxel-based morphometry study. Am. J. Neuroradiol. 28, 1859-1864. doi: 10.3174/ajnr.A0696

Badre, D., and Wagner, A. D. (2007). Left ventrolateral prefrontal cortex and the cognitive control of memory. Neuropsychologia 45, 2883-2901. doi: 10.1016/j.neuropsychologia.2007.06.015

Balser, N., Lorey, B., Pilgramm, S., Naumann, T., Kindermann, S., Stark, R., et al. (2014). The influence of expertise on brain activation of the action observation network during anticipation of tennis and volleyball serves. Front. Hum. Neurosci. 8:568. doi: 10.3389/fnhum.2014. 00568

Bangert, M., Peschel, T., Schlaug, G., Rotte, M., Drescher, D., and Hinrichs, H., et al. (2006). Shared networks for auditory and motor processing in professional pianists: evidence from fmri conjunction. Neuroimage 30, 917-926. doi: 10.1016/j.neuroimage.2005.10.044

Barbas, H., and Pandya, D. N. (1987). Architecture and frontal cortical connections of the premotor cortex (area 6) in the rhesus monkey. J. Comp. Neurol. 256, 211-228. doi: 10.1002/cne. 902560203

Baumann, S., Koeneke, S., Schmidt, C. F., Meyer, M., Lutz, K., and Jancke, L. (2007). A network for audio-motor coordination in skilled pianists and non-musicians. Brain Res. 1161, 65-78. doi: 10.1016/j.brainres.2007.05.045

Berkowitz, A. L., and Ansari, D. (2010). Expertise-related deactivation of the right temporoparietal junction during musical improvisation. Neuroimage 49, 712-719. doi: 10.1016/j.neuroimage.2009.08.042

Bermudez, P., Lerch, J. P., Evans, A. C., and Zatorre, R. J. (2008). Neuroanatomical correlates of musicianship as revealed by cortical thickness and voxel-based morphometry. Cereb. Cortex 19, 1583-1596. doi: 10.1093/cercor/bhn196

Bishop, D. T., Wright, M. J., Jackson, R. C., and Abernethy, B. (2013). Neural bases for anticipation skill in soccer: an fMRI study. J. Sport Exerc. Psychol. 35, 98-109. doi: $10.1123 /$ jsep.35.1.98
Science Research Project of Jiangsu Higher Education Institutions (No. 18KJB416001), Project of Six Talent Peaks of Jiangsu Province (No. SWYY-017), Shenzhen Fundamental Research Project (No. JCYJ20170412111316339), ShenzhenHong Kong Institute of Brain Science-Shenzhen Fundamental Research Institutions (No. 2019SHIBS003), Shenzhen Talent Peacock Plan (No. 827-000083), and Shanghai Sailing Program (No. 19YF1419000).

Boyke, J., Driemeyer, J., Gaser, C., Büchel, C., and May, A. (2008). Training induced brain structure changes in the elderly. J. Neurosci. 28, 7031-7035. doi: 10.1523/JNEUROSCI.0742-08.2008

Brouwer, R. M., Panizzon, M. S., Glahn, D. C., Hibar, D. P., Hua, X., Jahanshad, N., et al. (2017). Genetic influences on individual differences in longitudinal changes in global and subcortical brain volumes: results of the ENIGMA plasticity working group. Hum. Brain Mapp. 38, 4444-4458. doi: 10.1002/hbm.23672

Bzdok, D., Schilbach, L., Vogeley, K., Schneider, K., Laird, A. R., Langner, R., et al. (2012). Parsing the neural correlates of moral cognition: ALE meta-analysis on morality, theory of mind, and empathy. Brain Struct. Func. 217, 783-796. doi: 10.1007/s00429-012-0380-y

Caspers, S., Zilles, K., Laird, A. R., and Eickhoff, S. B. (2010). ALE meta-analysis of action observation and imitation in the human brain. NeuroImage 50, 1148-1167. doi: 10.1016/j.neuroimage.2009.12.112

Chang, Y. (2014). Reorganization and plastic changes of the human brain associated with skill learning and expertise. Front. Hum. Neurosci. 8:35. doi: 10.3389/fnhum.2014.00035

Chang, Y., Lee, J. J., Seo, J. H., Song, H. J., Kim, Y. T., Lee, H. J., et al. (2011). Neural correlates of motor imagery for elite archers. NMR Biomed. 24, 366-372. doi: $10.1002 / \mathrm{nbm} .1600$

Cheng, Y., Lin, C. P., Liu, H. L., Hsu, Y. Y., Lim, K. E., Hung, D., et al. (2007) Expertise modulates the perception of pain in others. Curr. Biol. 17, 1708-1713. doi: 10.1016/j.cub.2007.09.020

Courtney, S. M., Petit, L., Maisog, J. M., Ungerleider, L. G., and Haxby, J. V. (1998). An area specialized for spatial working memory in human frontal cortex. Science 279, 1347-1351. doi: 10.1126/science.279.5355.1347

de Lange, A. M. G., Bråthen, A. C. S., Rohani, D. A., Grydeland, H., Fjell, A. M., and Walhovd, K. B. (2017). The effects of memory training on behavioral and microstructural plasticity in young and older adults. Hum. Brain Mapp. 38, 5666-5680. doi: 10.1002/hbm.23756

Di, X., Zhu, S., Jin, H., Wang, P., Ye, Z., Zhou, K., et al. (2012). Altered resting brain function and structure in professional badminton players. Brain Connect. 2, 225-233. doi: 10.1089/brain.2011.0050

Dong, M., Li, J., Shi, X., Gao, S., Fu, S., Liu, Z., et al. (2015). Altered baseline brain activity in experts measured by amplitude of low frequency fluctuations (ALFF): a resting state fMRI study using expertise model of acupuncturists. Front. Hum. Neurosci. 9:99. doi: 10.3389/fnhum.2015.00099

Dong, M., Qin, W., Zhao, L., Yang, X., Yuan, K., Zeng, F., et al. (2014). Expertise modulates local regional homogeneity of spontaneous brain activity in the resting brain: an fMRI study using the model of skilled acupuncturists. Hum. Brain Mapp. 35, 1074-1084. doi: 10.1002/hbm.22235

Dong, M., Zhao, L., Yuan, K., Zeng, F., Sun, J., and Liu, J., et al. (2013). Length of acupuncture training and structural plastic brain changes in professional acupuncturists. PLoS ONE 8:e66591. doi: 10.1371/journal.pone.0066591

Duan, X., Liao, W., Liang, D., Qiu, L., Gao, Q., Liu, C., et al. (2012). Largescale brain networks in board game experts: insights from a domain-related task and task-free resting state. PLoS ONE 7:e32532. doi: 10.1371/journal.pone. 0032532

Duan, X., Long, Z., Chen, H., Liang, D., Qiu, L., Huang, X., et al. (2014). Functional organization of intrinsic connectivity networks in Chinese-chess experts. Brain Res. 1558, 33-43. doi: 10.1016/j.brainres.2014.02.033

Elder, G. J., and Taylor, J. P. (2014). Transcranial magnetic stimulation and transcranial direct current stimulation: treatments for cognitive and neuropsychiatric symptoms in the neurodegenerative dementias? Alzheimer's Res. Ther. 6:74. doi: 10.1186/s13195-014-0074-1 
Elmer, S., Hänggi, J., and Jäncke, L. (2014). Processing demands upon cognitive, linguistic, and articulatory functions promote grey matter plasticity in the adult multilingual brain: insights from simultaneous interpreters. Cortex 54, 179-189. doi: 10.1016/j.cortex.2014.02.014

Elmer, S., Meyer, M., and Jancke, L. (2010). Simultaneous interpreters as a model for neuronal adaptation in the domain of language processing. Brain Res. 1317, 147-156. doi: 10.1016/j.brainres.2009.12.052

Falk, E. B., and Bassett, D. S. (2017). Brain and social networks: fundamental building blocks of human experience. Trends Cogn. Sci. 21, 674-690. doi: 10.1016/j.tics.2017.06.009

Fauvel, B., Groussard, M., Chételat, G., Fouquet, M., Landeau, B., Eustache, F., et al. (2014). Morphological brain plasticity induced by musical expertise is accompanied by modulation of functional connectivity at rest. Neuroimage 90, 179-188. doi: 10.1016/j.neuroimage.2013.12.065

Feng, Y., Song, J., Yan, W., Wang, J., Zhao, C., and Zeng, Q. (2020). “Investigation of local white matter properties in professional chess player: a diffusion magnetic resonance imaging study based on automatic annotation fiber clustering," in IEEE Transactions on Cognitive and Developmental Systems. doi: 10.1109/TCDS.2020.2968116

Freund, H. J. (1990). Premotor area and preparation of movement. Revue Neurol. 146, 543-547.

Fuelscher, I., Caeyenberghs, K., Enticott, P. G., Williams, J., Lum, J., and Hyde, C. (2018). Differential activation of brain areas in children with developmental coordination disorder during tasks of manual dexterity: an ale meta-analysis. Neurosci. Biobehav. Rev. 86, 77-84. doi: 10.1016/j.neubiorev.2018.01.002

Fulton, J. F. (1935). A note on the definition of the "motor" and "premotor" areas. Brain 58, 311-316. doi: 10.1093/brain/58.2.311

Gage, F. H. (2002). Neurogenesis in the adult brain. J. Neurosci. 22, 612-613. doi: 10.1523/JNEUROSCI.22-03-00612.2002

Gaser, C., and Schlaug, G. (2003). Brain structures differ between musicians and non-musician. J. Neurosci. 23, 9240-9245. doi: 10.1523/JNEUROSCI.23-27-09240.2003

Genovese, C. R., Lazar, N. A., and Nichols, T. (2002). Thresholding of statistical maps in functional neuroimaging using the false discovery rate. Neuroimage 15, 870-878. doi: 10.1006/nimg.2001.1037

Ghahremani, D. G., Monterosso, J., Jentsch, J. D., Bilder, R. M., and Poldrack, R. A. (2009). Neural components underlying behavioral flexibility in human reversal learning. Cereb. Cortex 20, 1843-1852. doi: 10.1093/cercor/bhp247

Goghari, V. M., and MacDonald, A. W. III. (2009). The neural basis of cognitive control: response selection and inhibition. Brain Cogn. 71, 72-83. doi: 10.1016/j.bandc.2009.04.004

Greenlee, J. D., Oya, H., Kawasaki, H., Volkov, I. O., Severson, M. A. III., Howard, A. 3rd, et al. (2007). Functional connections within the human inferior frontal gyrus. J. Comp. Neurol. 503, 550-559. doi: 10.1002/cne.21405

Groussard, M., La Joie, R., Rauchs, G., Landeau, B., Chetelat, G., Viader, F., et al. (2010). When music and long-term memory interact: effects of musical expertise on functional and structural plasticity in the hippocampus. PLoS ONE 5:e13225. doi: 10.1371/journal.pone.0013225

Groussard, M., Viader, F., Landeau, B., Desgranges, B., Eustache, F., and Platel, H. (2014). The effects of musical practice on structural plasticity: the dynamics of grey matter changes. Brain Cogn. 90, 174-180. doi: 10.1016/j.bandc.2014.06.013

Habeck, C., Eich, T. S., Gu, Y., and Stern, Y. (2019). Occupational patterns of structural brain health: independent contributions beyond age, gender, intelligence, and age. Front. Hum. Neurosci. 13:449. doi: 10.3389/fnhum.2019.00449

Haglund, L., and Henriksson, C. (2003). Concepts in occupational therapy in relation to the ICF. Occup. Ther. Int. 10, 253-268. doi: 10.1002/oti.189

Hänggi, J. (2010). Structural neuroplasticity in the sensorimotor network of professional female ballet dancers. Hum. Brain Mapp. 31, 1196-1206. doi: $10.1002 / \mathrm{hbm} .20928$

Harris, R., and de Jong, B. M. (2014). Cerebral activations related to auditiondriven performance imagery in professional musicians. PLOS ONE 9:e93681. doi: 10.1371/journal.pone.0093681

Haslinger, B., Erhard, P., Altenmüller, E., Hennenlotter, A., Schwaiger, M., Gräfin von Einsiedel, H., et al. (2004). Reduced recruitment of motor association areas during bimanual coordination in concert pianists. Hum. Brain Mapp. 22, 206-215. doi: 10.1002/hbm.20028
Hernández, S. E., Suero, J., Barros, A., González-Mora, J. L., and Rubia, K. (2016). Increased grey matter associated with long-term sahaja yoga meditation: a voxel-based morphometry study. PLoS ONE 11:e0150757. doi: 10.1371/journal.pone.0150757

Hervais-Adelman, A., Moser-Mercer, B., and Golestani, N. (2015). Brain functional plasticity associated with the emergence of expertise in extreme language control. Neuroimage 114, 264-274. doi: 10.1016/j.neuroimage.2015.03.072

Hervais-Adelman, A., Moser-Mercer, B., Michel, C. M., and Golestani, N. (2014). fMRI of simultaneous interpretation reveals the neural basis of extreme language control. Cereb. Cortex 25, 4727-4739. doi: 10.1093/cercor/bhu158

Hocking, C. (2000). Occupational science: a stock take of accumulated insights. J. Occup. Sci. 7, 58-67. doi: 10.1080/14427591.2000.9686466

Hocking, C. (2009). The challenge of occupation: describing the things people do. J. Occup. Sci. 16, 140-150. doi: 10.1080/14427591.2009.9686655

Hu, J., Jiang, H., Liang, H., and Yang, H. (2018). Change of cerebral structural plasticity of track athletes based on magnetic resonance imaging. NeuroQuantology 16, 758-762. doi: 10.14704/nq.2018.16.6.1647

Huang, R., Lu, M., Song, Z., and Wang, J. (2015). Long-term intensive training induced brain structural changes in world class gymnasts. Brain Struct. Func. 220, 625-644. doi: 10.1007/s00429-013-0677-5

Huang, Z., Davis, H., Wolff, A., and Northoff, G. (2017). Thalamo-sensorimotor functional connectivity correlates with world ranking of olympic, elite, and high performance athletes. Neural Plast. 2017:1473783. doi: 10.1155/2017/1473783

Hüfner, K., Binetti, C., Hamilton, D. A., Stephan, T., Flanagin, V. L., Linn, J., et al. (2011). Structural and functional plasticity of the hippocampal formation in professional dancers and slackliners. Hippocampus 21, 855-865. doi: 10.1002/hipo.20801

Ito, M. (1993). Movement and thought: identical control mechanisms by the cerebellum. Trends Neurosci. 16, 448-450. doi: 10.1016/0166-2236(93) 90073-U

James, C. E., Oechslin, M. S., Van De Ville, D., Hauert, C. A., Descloux, C., and Lazeyras, F. (2014). Musical training intensity yields opposite effects on grey matter density in cognitive versus sensorimotor networks. Brain Struct. Func. 219, 353-366. doi: 10.1007/s00429-013-0504-Z

Jäncke, L., Koeneke, S., Hoppe, A., Rominger, C., and Hänggi, J. (2009). The architecture of the golfer's brain. PLoS ONE 4:e4785. doi: 10.1371/journal.pone.0004785

Jiang, J., Zhu, W., Shi, F., Liu, Y., Li, J., Qin, W., et al. (2009). Thick visual cortex in the early blind. J. Neurosci. 29, 2205-2211. doi: 10.1523/JNEUROSCI.5451-08.2009

Johansen-Berg, H., and Duzel, E. (2016). Neuroplasticity: effects of physical and cognitive activity on brain structure and function. NeuroImage 131, 1-3. doi: 10.1016/j.neuroimage.2016.03.081

Kawabata, H., and Zeki, S. (2004). Neural correlates of beauty. J. Neurophysiol. 91, 1699-1705. doi: 10.1152/jn.00696.2003

Kempermann, G. (2006). Adult Neurogenesis: Stem Cells and Neuronal Development in the Adult Brain. Oxford University Press.

Kempermann, G., Kuhn, H. G., and Gage, F. H. (1997). More hippocampal neurons in adult mice living in an enriched environment. Nature 386, 493-5. doi: $10.1038 / 386493 \mathrm{a} 0$

Kim, Y. T., Seo, J. H., Song, H. J., Yoo, D. S., Lee, H. J., and Lee, J., et al. (2011). Neural correlates related to action observation in expert archers. Behav. Brain Res. 223, 342-347. doi: 10.1016/j.bbr.2011.04.053

Kirk, U., Skov, M., Christensen, M. S., and Nygaard, N. (2009). Brain correlates of aesthetic expertise: a parametric fMRI study. Brain Cogn. 69, 306-315. doi: 10.1016/j.bandc.2008.08.004

Kleber, B., Veit, R., Moll, C. V., Gaser, C., Birbaumer, N., and Lotze, M. (2016). Voxel-based morphometry in opera singers: increased gray-matter volume in right somatosensory and auditory cortices. Neuroimage 133, 477-483. doi: 10.1016/j.neuroimage.2016.03.045

Koeneke, S., Lutz, K., Wüstenberg, T., and Jäncke, L. (2004). Long-term training affects cerebellar processing in skilled keyboard players. Neuroreport 15, 1279-1282. doi: 10.1097/01.wnr.0000127463.10147.e7

Kramer, A. F., Bherer, L., Colcombe, S. J., Dong, W., and Greenough, W. T. (2004). Environmental influences on cognitive and brain plasticity during aging. $J$. Gerontol. A Biol. Sci. Med. Sci. 59, M940-M957. doi: 10.1093/gerona/59. 9.M940 
Krawczyk, D. C., Boggan, A. L., McClelland, M. M., and Bartlett, J. C. (2011). The neural organization of perception in chess experts. Neurosci. Lett. 499, 64-69. doi: 10.1016/j.neulet.2011.05.033

Laird, A. R., Eickhoff, S. B., Kurth, F., Fox, P. M., Uecker, A. M., Turner, J. A., et al. (2009). ALE meta-analysis workflows via the brainmap database: progress towards a probabilistic functional brain atlas. Front. Neuroinform. 3:23. doi: 10.3389/neuro.11.023.2009

Laird, A. R., Fox, P. M., Price, C. J., Glahn, D. C., Uecker, A. M., Lancaster, J. L., et al. (2005). ALE meta-analysis: controlling the false discovery rate and performing statistical contrasts. Hum. Brain Mapp. 25, 155-164. doi: 10.1002/hbm.20136

Lee, H., and Noppeney, U. (2011). Long-term music training tunes how the brain temporally binds signals from multiple senses. Proc. Natl. Acad. Sci. U.S.A. 108, E1441-E1450. doi: 10.1073/pnas.1115267108

Leiner, H. C., Leiner, A. L., and Dow, R. S. (1993). Cognitive and language functions of the human cerebellum. Trends Neurosci. 16, 444-447. doi: 10.1016/0166-2236(93)90072-T

Li, G., Li, X., Luo, C., Yao, D. (2018). Modern dance training and string instrument training have different effects on grey matter architecture. Adv. Psychol. Sci. 26, 1165-1173. doi: 10.3724/SP.J.1042.2018.01165

Li, J., Lai, Y., Luo, C., Yao, L., and Yao, D. (2011). Differences in brain structures between female musicians and non-musicians. Int. J. Bioelectromag. $13,274-277$

Li, K., Jiang, J., Qiu, L., Yang, X., Huang, X., Lui, S., et al. (2015). A multimodal MRI dataset of professional chess players. Sci. Data 2:150044. doi: 10.1038/sdata.2015.44

Liu, S., Sheng, J., Li, B., and Zhang, X. (2017). Recent advances in non-invasive brain stimulation for major depressive disorder. Front. Hum. Neurosci. 11:526. doi: 10.3389/fnhum.2017.00526

Lotze, M., Scheler, G., Tan, H. R., Braun, C., and Birbaumer, N. (2003). The musician's brain: functional imaging of amateurs and professionals during performance and imagery. Neuroimage 20, 1817-1829. doi: 10.1016/j.neuroimage.2003.07.018

Lövdén, M., Bäckman, L., Lindenberger, U., Schaefer, S., and Schmiedek, F. (2010). A theoretical framework for the study of adult cognitive plasticity. Psychol. Bull. 136, 659-76. doi: 10.1037/a0020080

Lu, M. T., Preston, J. B., and Strick, P. L. (1994). Interconnections between the prefrontal cortex and the premotor areas in the frontal lobe. J. Comp. Neurol. 341, 375-392. doi: 10.1002/cne.903410308

Lu, Y., Zhao, Q., Wang, Y., and Zhou, C. (2018). Ballroom dancing promotes neural activity in the sensorimotor system: a resting-state fMRI study. Neural Plast. 2018:2024835. doi: 10.1155/2018/2024835

Luo, C., Guo, Z. W., Lai, Y. X., Liao, W., Liu, Q., Kendrick, K. M., et al. (2012). Musical training induces functional plasticity in perceptual and motor networks: insights from resting-state FMRI. PLoS ONE 7:e36568. doi: 10.1371/journal.pone.0036568

Luppino, G., Matelli, M., Camarda, R., and Rizzolatti, G. (1993). Corticocortical connections of area F3 (SMA-proper) and area F6 (pre-SMA) in the macaque monkey. J. Comp. Neurol. 338, 114-140. doi: 10.1002/cne.9033 80109

Maguire, E. A., Gadian, D. G., Johnsrude, I. S., Good, C. D., Ashburner, J., Frackowiak, R. S., et al. (2000). Navigation-related structural change in the hippocampi of taxi drivers. Proc. Natl. Acad. Sci. U.S.A. 97, 4398-4403. doi: $10.1073 /$ pnas.070039597

Maguire, E. A., Spiers, H. J., Good, C. D., Hartley, T., Frackowiak, R. S., and Burgess, N. (2003). Navigation expertise and the human hippocampus: a structural brain imaging analysis. Hippocampus 13, 250-259. doi: 10.1002/hipo.10087

Mahncke, H. W., Bronstone, A., and Merzenich, M. M. (2006). Brain plasticity and functional losses in the aged: scientific bases for a novel intervention. Progr. Brain Res. 157, 81-109. doi: 10.1016/S0079-6123(06)57006-2

Maldjian, J. A., Laurienti, P. J., Kraft, R. A., and Burdette, J. H. (2003). An automated method for neuroanatomic and cytoarchitectonic atlasbased interrogation of fMRI data sets. Neuroimage 19, 1233-1239. doi: 10.1016/S1053-8119(03)00169-1

Mayeli, M., Rahmani, F., and Aarabi, M. H. (2018). Comprehensive investigation of white matter tracts in professional chess players and relation to expertise: region of interest and DMRI connectometry. Front. Neurosci. 12:288 doi: 10.3389/fnins.2018.00288

Messé, A., Rudrauf, D., Benali, H., and Marrelec, G. (2014). Relating structure and function in the human brain: relative contributions of anatomy, stationary dynamics, and non-stationarities. PLoS Comput. Biol. 10:e1003530. doi: 10.1371/journal.pcbi.1003530

Middleton, F. A., and Strick, P. L. (1994). Anatomical evidence for cerebellar and basal ganglia involvement in higher cognitive function. Science 266, 458-461. doi: $10.1126 /$ science. 7939688

Moher, D., Liberati, A., Tetzlaff, J., and Altman, D. G. (2010). Preferred reporting items for systematic reviews and meta-analyses: the PRISMA statement. Int. J. Surg. 8, 336-341. doi: 10.1016/j.ijsu.2010.02.007

Molnar-Szakacs, I., and Overy, K. (2006). Music and mirror neurons: from motion to'e'motion. Soc. Cogn. Affect. Neurosci. 1, 235-241. doi: 10.1093/scan/nsl029

Müller, V. I., Cieslik, E. C., Laird, A. R., Fox, P. T., Radua, J., Mataix-Cols, D., et al. (2018). Ten simple rules for neuroimaging meta-analysis. Neurosc. Biobehav. Rev. 84, 151-161. doi: 10.1016/j.neubiorev.2017.11.012

Neumann, N., Domin, M., Erhard, K., and Lotze, M. (2018). Voxel-based morphometry in creative writers: grey matter increase in a prefronto-thalamiccerebellar network. Eur. J. Neurosci. 48, 1647-1653. doi: 10.1111/ejn.13952

Obusez, E. C., Lowe, M., Oh, S. H., Wang, I., Bullen, J., Ruggieri, P., et al. (2018). 7T MR of intracranial pathology: preliminary observations and comparisons to $3 \mathrm{~T}$ and $1.5 \mathrm{~T}$. Neuroimage 168, 459-476. doi: 10.1016/j.neuroimage.2016. 11.030

Park, H. J., and Friston, K. (2013). Structural and functional brain networks: from connections to cognition. Science 342:1238411. doi: 10.1126/science.1238411

Pascual-Leone, A., Amedi, A., Fregni, F., and Merabet, L. B. (2005). The plastic human brain cortex. Annu. Rev. Neurosci. 28, 377-401. doi: 10.1146/annurev.neuro.27.070203.144216

Pascual-Leone, A., Davey, N. J., Rothwell, J., Wasserman, E. M., and Puri, B. K. (Eds.). (2002). Handbook of Transcranial Magnetic Stimulation. Arnold.

Paulus, W. (2011). Transcranial electrical stimulation (tES-tDCS; tRNS, tACS) methods. Neuropsychol. Rehabil. 21, 602-617. doi: 10.1080/09602011.2011.557292

Pazart, L., Comte, A., Magnin, E., Millot, J. L., and Moulin, T. (2014). An fMRI study on the influence of sommeliers' expertise on the integration of flavor. Front. Behav. Neurosci. 8:358. doi: 10.3389/fnbeh.2014.00358

Peng, L., Zeng, L. L., Liu, Q., Wang, L., Qin, J., Xu, H., et al. (2018). Functional connectivity changes in the entorhinal cortex of taxi drivers. Brain Behav. 8:e01022. doi: 10.1002/brb3.1022

Peyron, R., Laurent, B., and Garcia-Larrea, L. (2000). Functional imaging of brain responses to pain. A review and meta-analysis. Neurophysiol. Clin. Clin. Neurophysiol. 30, 263-288. doi: 10.1016/S0987-7053(00)00227-6

Picard, N., and Strick, P. L. (2001). Imaging the premotor areas. Curr. Opin. Neurobiol. 11, 663-672. doi: 10.1016/S0959-4388(01)00266-5

Pilgramm, S., Lorey, B., Stark, R., Munzert, J., Vaitl, D., and Zentgraf, K. (2010). Differential activation of the lateral premotor cortex during action observation. BMC Neurosci. 11:89. doi: 10.1186/1471-2202-11-89

Popescu, T., Sader, E., Schaer, M., Thomas, A., Terhune, D. B., Dowker, A., et al. (2019). The brain-structural correlates of mathematical expertise. Cortex 114, 140-150. doi: 10.1016/j.cortex.2018.10.009

Read, H., Roush, S., and Downing, D. (2018). Early intervention in mental health for adolescents and young adults: a systematic review. Am. J. Occup. Ther. 72, 7205190040p1-7205190040p8. doi: 10.5014/ajot.2018.033118

Reis, J., Robertson, E., Krakauer, J. W., Rothwell, J., Marshall, L., Gerloff, C., et al. (2008). Consensus: can tDCS and TMS enhance motor learning and memory formation? Brain Stimul. 1, 363-369. doi: 10.1016/j.brs.2008.08.001

Rizzolatti, G., Cattaneo, L., Fabbri-Destro, M., and Rozzi, S. (2014). Cortical mechanisms underlying the organization of goal-directed actions and mirror neuron-based action understanding. Physiol. Rev. 94, 655-706. doi: 10.1152/physrev.00009.2013

Schneider, P., Scherg, M., Dosch, H. G., Specht, H. J., Gutschalk, A., and Rupp, A. (2002). Morphology of heschl's gyrus reflects enhanced activation in the auditory cortex of musicians. Nat. Neurosci. 5, 688-94. doi: 10.1038/nn871

Schubotz, R. I., and von Cramon, D. (2003). Functional-anatomical concepts of human premotor cortex: evidence from fMRI and PET studies. Neuroimage 20, S120-31. doi: 10.1016/j.neuroimage.2003.09.014 
Schulze, K., Zysset, S., Mueller, K., Friederici, A. D., and Koelsch, S. (2011). Neuroarchitecture of verbal and tonal working memory in nonmusicians and musicians. Hum. Brain Mapp. 32, 771-783. doi: 10.1002/hbm.21060

Seo, J., Kim, Y. T., Song, H. J., Lee, H. J., Lee, J., Jung, T. D., et al. (2012). Stronger activation and deactivation in archery experts for differential cognitive strategy in visuospatial working memory processing. Behav. Brain Res. 229, 185-193. doi: 10.1016/j.bbr.2012.01.019

Sharot, T., Kanai, R., Marston, D., Korn, C. W., Rees, G., and Dolan, R. J. (2012). Selectively altering belief formation in the human brain. Proc. Natl. Acad. Sci. U.S.A. 109, 17058-17062. doi: 10.1073/pnas.1205828109

Shaw, P., Eckstrand, K., Sharp, W., Blumenthal, J., Lerch, J. P., Greenstein, D. E. E. A., et al. (2007). Attention-deficit/hyperactivity disorder is characterized by a delay in cortical maturation. Proc. Natl. Acad. Sci. U.S.A. 104, 19649-19654. doi: 10.1073/pnas.0707741104

Shen, H., Li, Z., Qin, J., Liu, Q., Wang, L., Zeng, L. L., et al. (2016). Changes in functional connectivity dynamics associated with vigilance network in taxi drivers. Neuroimage 124, 367-378. doi: 10.1016/j.neuroimage.2015.09.010

Sluming, V., Barrick, T., Howard, M., Cezayirli, E., Mayes, A., and Roberts, N. (2002). Voxel-based morphometry reveals increased gray matter density in Broca's area in male symphony orchestra musicians. Neuroimage 17, 1613-1622. doi: 10.1006/nimg.2002.1288

Song, L., Peng, Q., Liu, S., and Wang, J. (2019). Changed hub and functional connectivity patterns of the posterior fusiform gyrus in chess experts. Brain Imaging Behav. 14, 797-805. doi: 10.1007/s11682-018-0020-0

Sowell, E. R., Thompson, P. M., Leonard, C. M., Welcome, S. E., Kan, E., and Toga, A. W. (2004). Longitudinal mapping of cortical thickness and brain growth in normal children. J. Neurosci. 24, 8223-8231. doi: 10.1523/JNEUROSCI.1798-04.2004

Sreenivasan, K., Zhuang, X., Banks, S. J., Mishra, V., Yang, Z., Deshpande, G., et al. (2017). Olfactory network differences in master sommeliers: connectivity analysis using granger causality and graph theoretical approach. Brain Connect. 7, 123-136. doi: 10.1089/brain.2016.0458

Tan, X. Y., Pi, Y. L., Wang, J., Li, X. P., Zhang, L. L., Dai, W., et al. (2017). Morphological and functional differences between athletes and novices in cortical neuronal networks. Front. Hum. Neurosci. 10:660. doi: $10.3389 /$ fnhum. 2016.00660

Tanaka, S., Honda, M., and Sadato, N. (2005). Modality-specific cognitive function of medial and lateral human Brodmann area 6. J. Neurosci. 25, 496-501. doi: 10.1523/JNEUROSCI.4324-04.2005

Tanaka, S., Michimata, C., Kaminaga, T., Honda, M., and Sadato, N. (2002). Superior digit memory of abacus experts: an event-related functional MRI study. Neuroreport 13, 2187-2191. doi: 10.1097/00001756-200212030-00005

Tomasino, B., Maieron, M., Guatto, E., Fabbro, F., and Rumiati, R. I. (2013). How are the motor system activity and functional connectivity between the cognitive and sensorimotor systems modulated by athletic expertise? Brain Res. 1540, 21-41. doi: 10.1016/j.brainres.2013.09.048

Turkeltaub, P. E., Eden, G. F., Jones, K. M., and Zeffiro, T. A. (2002). Meta-analysis of the functional neuroanatomy of single-word reading: method and validation. Neuroimage 16, 765-780. doi: 10.1006/nimg.2002.1131

Turkeltaub, P. E., Eickhoff, S. B., Laird, A. R., Fox, M., Wiener, M., and Fox, P. (2012). Minimizing within-experiment and within-group effects in activation likelihood estimation meta-analyses. Hum. Brain Mapp. 33, 1-13. doi: 10.1002/hbm.21186

Vaquero, L., Hartmann, K., Ripollés, P., Rojo, N., Sierpowska, J., François, C., et al. (2016). Structural neuroplasticity in expert pianists depends on the age of musical training onset. Neuroimage 126, 106-119. doi: 10.1016/j.neuroimage.2015.11.008

Vartanian, O., and Goel, V. (2004). Neuroanatomical correlates of aesthetic preference for paintings. Neuroreport 15, 893-897. doi: 10.1097/00001756-200404090-00032

Wagner, A. P., Schmoll, H., Badan, I., Platt, D., and Kessler, C. (2000). Brain plasticity: to what extent do aged animals retain the capacity to coordinate gene activity in response to acute challenges. Exp. Gerontol. 35, 1211-1227. doi: 10.1016/S0531-5565(00)00154-6

Walhovd, K. B., Krogsrud, S. K., Amlien, I. K., Bartsch, H., Bjørnerud, A., Due-Tønnessen, P., et al. (2016). Neurodevelopmental origins of lifespan changes in brain and cognition. Proc. Natl. Acad. Sci. U.S.A. 113, 9357-9362. doi: $10.1073 /$ pnas. 1524259113
Wang, L., Liu, Q., Shen, H., Li, H., and Hu, D. (2015). Large-scale functional brain network changes in taxi drivers: evidence from resting-state fMRI. Hum. Brain Mapp. 36, 862-871. doi: 10.1002/hbm. 22670

Wang, N., Wu, H., Xu, M., Yang, Y., Chang, C., Zeng, W., et al. (2018). Occupational functional plasticity revealed by brain entropy: a resting-state fMRI study of seafarers. Hum. Brain Mapp. 39, 2997-3004. doi: 10.1002/hbm.24055

Wang, N., Zeng, W., Shi, Y., and Yan, H. (2017). Brain functional plasticity driven by career experience: a resting-state fMRI study of the seafarer. Front. Psychol. 8:1786. doi: 10.3389/fpsyg.2017.01786

Wei, G., Luo, J., and Li, Y. (2009). Brain structure in diving players on MR imaging studied with voxel-based morphometry. Progr. Nat. Sci. 19, 1397-1402. doi: $10.1016 /$ j.pnsc.2008.12.009

Wei, G., Zhang, Y., Jiang, T., and Luo, J. (2011). Increased cortical thickness in sports experts: a comparison of diving players with the controls. PLoS ONE 6:e17112. doi: 10.1371/journal.pone.0017112

Weiskopf, N., Suckling, J., Williams, G., Correia, M. M., Inkster, B., Tait, R., et al. (2013). Quantitative multi-parameter mapping of R1, PD*, MT, and R2* at 3T: a multi-center validation. Front. Neurosci. 7:95. doi: 10.3389/fnins.2013. 00095

Wise, S. P. (1985). The primate premotor cortex: past, present, and preparatory. Annu. Rev. Neurosci. 8, 1-19. doi: 10.1146/annurev.ne.08.030185.000245

Woollett, K., Spiers, H. J., and Maguire, E. A. (2009). Talent in the taxi: a model system for exploring expertise. Philos. Trans. R. Soc. B Biol. Sci. 364, 1407-1416. doi: $10.1098 /$ rstb.2008.0288

Wright, I. C., Rabe-Hesketh, S., Woodruff, P. W., David, A. S., Murray, R. M., and Bullmore, E. T. (2000). Meta-analysis of regional brain volumes in schizophrenia. Am. J. Psychiatry 157, 16-25. doi: 10.1176/ajp.157.1.16

Wright, M., Bishop, D. T., Jackson, R., and Abernethy, B. (2013). Brain regions concerned with the identification of deceptive soccer moves by higher-skilled and lower-skilled players. Front. Hum. Neurosci. 7:851. doi: 10.3389/fnhum.2013.00851

Wright, M. J., Bishop, D. T., Jackson, R. C., and Abernethy, B. (2011). Cortical fMRI activation to opponents' body kinematics in sport-related anticipation: expert-novice differences with normal and point-light video. Neurosci. Lett. 500, 216-221. doi: 10.1016/j.neulet.2011.06.045

Xu, H., Wang, P., Ye, Z. E., Di, X., Xu, G., Mo, L., et al. (2016). The role of medial frontal cortex in action anticipation in professional badminton players. Front. Psychol. 7:1817. doi: 10.3389/fpsyg.2016.01817

Zatorre, R. J. (2013). Predispositions and plasticity in music and speech learning: neural correlates and implications. Science 342, 585-589. doi: $10.1126 /$ science. 1238414

Zeng, W., Wang, N., Shi, Y., and Yan, H. (2016): Brain functional connectivity analysis and brain plasticity from perspective of fMRI technique. (in Chinese) J. Data Acquisit. Process. 31, 856-867. doi: 10.16337/j.1004-9037.2016.05.002

Zhang, J. X., Leung, H. C., and Johnson, M. K. (2003). Frontal activations associated with accessing and evaluating information in working memory: an fMRI study. Neuroimage 20, 1531-1539. doi: 10.1016/j.neuroimage.2003.07.016

Zhang, W., Zhao, F., Ma, L., and Qin, W. (2018). Altered Spontaneous regional brain activity in insula and visual areas in professional traditional Chinese Pingju opera actors. Front. Neurosci. 12:450. doi: 10.3389/fnins.2018. 00450

Zilles, K. (1992). Neuronal plasticity as an adaptive property of the central nervous system. Ann. Anat. Anatom. Anzeiger 174, 383-391. doi: 10.1016/S0940-9602(11)80255-4

Conflict of Interest: The authors declare that the research was conducted in the absence of any commercial or financial relationships that could be construed as a potential conflict of interest.

Copyright $\odot 2020 \mathrm{Wu}$, Yan, Yang, Xu, Shi, Zeng, Li, Zhang, Chang and Wang. This is an open-access article distributed under the terms of the Creative Commons Attribution License (CC BY). The use, distribution or reproduction in other forums is permitted, provided the original author(s) and the copyright owner(s) are credited and that the original publication in this journal is cited, in accordance with accepted academic practice. No use, distribution or reproduction is permitted which does not comply with these terms. 